\title{
HPVB and HPVZM shaped growth of CdZnTe, CdSe and ZnSe crystals
}

\author{
N. N. Kolesnikov**, R. B. James ${ }^{+}$, N. S. Berzigiarova ${ }^{*}$, M. P. Kulakov ${ }^{*}$ \\ $\left(^{*}\right)$ - Institute of Solid State Physics, Russian Academy of Sciences, Russia \\ $(+)$ - Brookhaven National Laboratory, Upton, NY, USA
}

\begin{abstract}
High-pressure Bridgman (HPVB) and vertical zone melting (HPVZM) growth processes have been applied for the manufacturing of $\mathrm{Cd}_{1-\mathrm{x}} \mathrm{Zn}_{\mathrm{x}} \mathrm{Te}(\mathrm{x}=0.04-0.2)$, CdSe and $\mathrm{ZnSe}$ crystal tapes with sizes up to $120 \times 120 \times 12 \mathrm{~mm}$. The influences of the technological parameters describing the growth processes on the crystal quality and some selected material properties are discussed. The dependence of the inclusion (bubbles) content on the deviation from melt stoichiometry is determined. A method for growing plates with low content of inclusions is described. High-resistivity crystal tapes of undoped CdZnTe $\left(10^{10} \mathrm{Ohm} \times \mathrm{cm}\right)$, CdSe $\left(10^{11} \mathrm{Ohm} \times \mathrm{cm}\right)$ and $\mathrm{ZnSe}\left(>10^{11} \mathrm{Ohm} \times \mathrm{cm}\right)$ were prepared. The possibility of tape growth on oriented seeds is shown for the example of CdSe. The primary differences between HPVB and HPVZM results are described. The main HPVZM advantage for II-VI compound crystal growth is the possibility of obtaining crystals with more stoichiometric composition or with a controlled deviation from stoichiometry. Hence, HPVZM is preferable for growing high-resistivity II-VI crystals with low inclusion content and possibly with better transport properties.
\end{abstract}

Keywords: Crystal growth, shaped crystal growth, ZnSe, CdSe, CdZnTe, CZT, HPVB, Bridgman, HPVZM, zone melting, radiation detectors.

\section{INTRODUCTION}

Growth of bulk II-VI compound crystals is often carried out by the high-pressure vertical Bridgman method (HPVB) or by means of vertical zone melting under high pressure of an inert gas (HPVZM). HPVB is the most widely applied technique, especially for the manufacturing of CdTe and CdZnTe detector-grade crystals. At the same time there is strong experimental evidence that HPVZM has distinct advantages compared to HPVB [1], because it allows for better control of the main composition of the crystal. In particular, authors of Ref. [1] reported preparation of $\mathrm{ZnSe}$ crystals with stoichiometric composition and with preset deviations from stoichiometry. It is relatively easy to grow $\mathrm{ZnSe}$ from the melt because, due to a self-compensation, the crystals always have high specific resistivity (about $10^{12}$ $\mathrm{Ohm} \times \mathrm{cm}$ ) and thus they are ready for many applications, such as IR optics and electrooptic modulators. Growth of some other II-VI compound crystals, especially CdTe, CdSe, CdS and their mixtures, presents more difficulty. This is because the specific resistivity of these materials tends to vary across a very wide range $\left(1 \times 10^{1}-1 \times 10^{12} \mathrm{Ohm} \times \mathrm{cm}\right)$ with variations of the growth process parameters. In addition, the tellurides can easily change their conductivity type with changing of the growth conditions.

This paper describes application of HPVB and HPVZM methods for CdZnTe, CdSe and ZnSe shaped crystal growth. ZnSe was used as a model material, because its properties are the most studied among the II-VI compounds. In addition, $\mathrm{ZnSe}$ is transparent in the visible spectral region and thus allows for a simple observation of many extended defects, such as bubbles and other secondary phases, and their distribution.

The difference between HPVB and HPVZM results is described. An approach to grow bubble-free crystals is discussed.

\footnotetext{
- Institute of Solid State Physics, Russian Acad. Sci., Chernogolovka, Moscow Distr., 142432 Russia. Tel: 7-(096)-52-22074; Fax: 7-(096)-52-49701; nkolesn@issp.ac.ru
} 


\section{COMPARISON OF HPVB AND HPVZM FOR GROWTH OF II-VI CRYSTALS}

It is known [1] that II-VI compounds have particular physical-chemical properties that present a challenge for the crystal growth. Zinc and cadmium chalcogenides are refractory, chemically corrosive (in liquid and vapor state) materials with high pressures of their own vapors at temperatures exceeding their respective melting point. While evaporating, II-VI compounds dissociate to the components; the metals evaporate in atomic form and chalcogens - in molecular one (in general case as $\mathrm{B}_{2}$, except the tellurium vapors at low temperatures). The typical crucible material graphite - is partially permeable for vapors of II-VI compounds and their components [1]. As a result, the composition of the melt shifts to an excess of one of the components due to a difference of the vapors diffusion coefficients in an external medium, which is an inert gas.

\subsection{The HPVB growth (in an inert gas atmosphere) of binary II-VI crystals in a graphite crucible}

Lets assume that:

- the mixing in the melt is complete;

- the solution of an excessive component in the melt is perfect;

- the equilibrium of the gas phase with the melt is achieved instantly;

- the content of the excessive component is low, so $P_{i}<<P_{g}$, where $P_{i}$ is the pressure of the excessive component above the II-VI melt $\left(i=\mathrm{A}^{\mathrm{II}}\right.$ or $\left.\mathrm{B}^{\mathrm{VI}}\right)$ and $P_{g}$ is the pressure of the inert gas.

In that case the mass balance of the melt crystallization with excess of the component $i$ at constant temperature and pressure is given by the following equation:

$$
\frac{d C_{i}}{d x}=\frac{C_{i}\left(1-k_{i}\right) S}{V}+\frac{C_{i}(A-E) F}{V v}-\frac{\left(E P_{0, i} / P_{0, i}^{0}-0.5 A\right) F}{V V_{m} v}
$$

where $C_{i}$ is the concentration of the $i$ component in the melt; $x$ is the current position of the crystallization front; $k_{i}$ is the segregation coefficient of the $i$ component; $V$ is the volume of the melt; $S$ is the area of the melt horizontal cross-section; $v$ is the growth rate; $P_{0, i}$ is the equilibrium vapor pressure of the $i$ component above the II-VI melt; $P_{0, i}^{0}$ is the equilibrium vapor pressure of the $i$ component above its own melt; $V_{m}$ is the molar volume of the II-VI melt; $F$ is the full area of the evaporation surface; $E$ is the specific evaporation flux of the pure excessive component in the given conditions; $A$ is the stationary rate of the evaporation of the other component of the melt.

In case of the $\mathrm{A}^{\mathrm{II}}$ excess in the melt:

$$
E=\delta D_{A^{I I}} P_{0, A^{I I}}^{0} V_{m} / R T \Delta \quad \text { and } \quad A=\delta D_{B^{V I}} P_{B^{V I}} V_{m} / R T \Delta
$$

and in case of $\mathrm{B}^{\mathrm{VI}}$ excess in the melt

$$
E=\delta D_{B^{V I}} P_{0, B^{V I}}^{0} V_{m} / R T \quad \text { and } \quad A=\delta D_{A^{I I}} P_{A^{I I}} V_{m} / R T \Delta
$$

where $\delta$ is the coefficient of the graphite permeability; $D_{i}$ is the diffusion coefficient of the $i$ component vapors in the inert gas; $R$ is the universal gas constant; $T$ is the temperature; $\Delta$ is the thickness of the graphite crucible wall ( $\delta$ is taken from [1]).

$$
E-A \approx\left(D_{A^{I I}} P_{0, A^{I I}}^{0} / R T \Delta\right) V_{m} \delta
$$

in case of the $\mathrm{A}^{\mathrm{II}}$ excess and

$$
E-A \approx\left(D_{B^{V I}} P_{0, B^{V I}}^{0} / R T \Delta\right) V_{m} \delta
$$

in case of the $\mathrm{B}^{\mathrm{VI}}$ excess.

Equation (1) allows solution in two different assumptions:

1. Evaporation of one of the components does not lead to additional shift in the stoichiometry;

2. Segregation coefficient for one of the components is equal to 1 , while the evaporation rate of another component is a constant. 
The first assumption means that the last member in equation (1) is equal to zero. It is then possible to solve Eq. (1) after setting the crystal length $L$ and determining a melt column height as $g=L-x$. Here,

$$
C_{i}=k_{i} C_{0, i}(1-g)^{k-1+(E-A) / v} \times e^{-f L(E-A) g / S v}
$$
the material.

where $f$ is the perimeter of the horizontal melt cross-section; $C_{0, i}$ is the start concentration of the $i$ component in

Equation (4) describes decreasing $C_{i}$ down to $C_{i}=0$, but it is obvious that at any starting concentration and with any technological parameters the $C_{i}=0$ situation appears only in the end of the crystal, i.e., there is no refining of the material from the excessive component if $C_{i}>0$ in the initial load.

The second assumption gives the possibility to consider a change in the melt volume due to evaporation. In this case the solution of Eq. (1) is:

$$
\left(C_{i}-C_{i, q s}\right)\left(C_{i}-C_{i, q s)}=\exp \left(-\frac{t f}{S} \delta D_{i} P_{0, i}^{0} V_{m} / R T \Delta\right)\right.
$$

where $t$ is the time, and $C_{i, q s}$ is the quasi-stationary concentration of the excessive component. It is "quasistationary", because the equation allows only asymptotic approach to it and the concentration coincides with $C_{i, q s}$ exactly only when $t=(S / A f) \ln (F / S)$, i.e., in the final moment of the evaporation itself.

is:

The equation that describes changing of the main composition of the II-VI compound in the HPVB conditions

$$
\frac{C_{i}-C_{i, q s}}{C_{i, 0}-C_{i, q s}}=(1-g)^{(E-A) / v} \exp \left[-\frac{(E-A) L f g}{v S}\right]
$$

We compared the results of calculations using Eq. (6) with the experimental data on the main composition of ZnSe crystals, as grown by HPVB in an Ar atmosphere with different technological parameters (see fig. 1 and table 1). As it was already mentioned, $\mathrm{ZnSe}$ is convenient as a model compound, because it was possible to obtain data on $\mathrm{ZnSe}$ properties, which are necessary for calculating Eqs. [1 - 3].

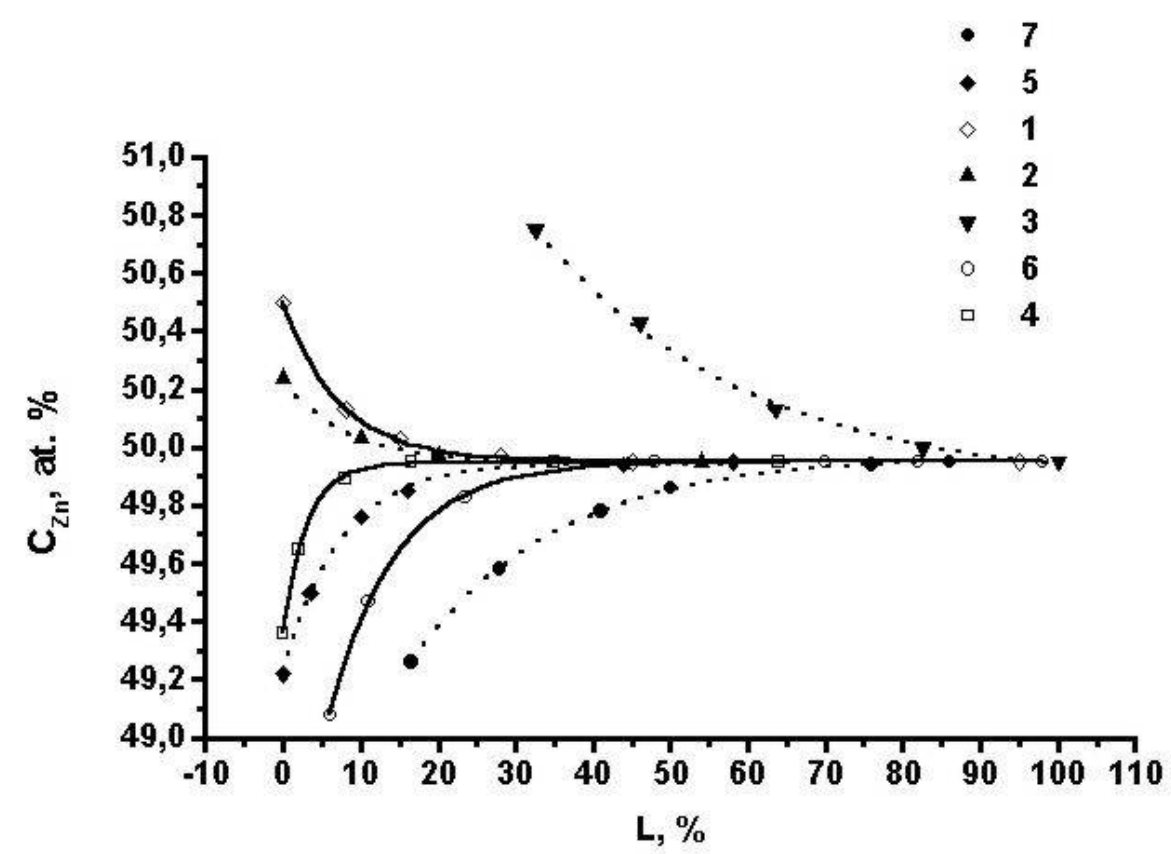

Fig. 1. Changing of the main composition (in Zn content) along the HPVB grown ZnSe crystals. The curves are calculated, and the points represent the experimental data. The conditions of the experiments are given in Table 1. Here, the numbers of the curves correspond to the row numbers in Table 1. 
Table 1. Condition of the experiments for ZnSe HPVB growth.

\begin{tabular}{|c|c|c|c|c|c|c|c|c|}
\hline $\begin{array}{l}\text { No of } \\
\text { experiment }\end{array}$ & $\nu, \mu \mathrm{m} / \mathrm{s}$ & $\begin{array}{l}E-A, \\
\mu \mathrm{m} / \mathrm{s}\end{array}$ & $L, \mathrm{~m}$ & $f, \mathrm{~m}$ & $\mathrm{~S}, \mathrm{~m}^{2}$ & $\begin{array}{l}C_{0, Z n}, \\
\text { at. } \%\end{array}$ & $\Delta, \mathrm{m}$ & Notes \\
\hline 1 & 2.78 & 2.30 & 0.12 & 0.1256 & $1.1256 \times 10^{-3}$ & 50.5 & $2 \times 10^{-3}$ & \multirow{7}{*}{$\begin{array}{l}\text { In all } \\
\text { experiments } \\
P_{g}=2.0 \mathrm{MPa} \\
\text { (Ar), } \\
\mathrm{T}=1800 \mathrm{~K}, \\
\delta=0.033\end{array}$} \\
\hline 2 & 2.78 & 2.30 & 0.12 & 0.1256 & $1.1256 \times 10^{-3}$ & 50.24 & $3 \times 10^{-3}$ & \\
\hline 3 & 19.50 & 3.45 & 0.08 & 0.1067 & $0.9070 \times 10^{-3}$ & 52.00 & $2 \times 10^{-3}$ & \\
\hline 4 & 2.78 & 5.93 & 0.12 & 0.1256 & $1.1256 \times 10^{-3}$ & 49.36 & $3 \times 10^{-3}$ & \\
\hline 5 & 5.56 & 5.93 & 0.10 & 0.1256 & $1.1256 \times 10^{-3}$ & 49.77 & $3 \times 10^{-3}$ & \\
\hline 6 & 5.56 & 5.93 & 0.08 & 0.1256 & $1.1256 \times 10^{-3}$ & 49.22 & $3 \times 10^{-3}$ & \\
\hline 7 & 19.50 & 5.93 & 0.12 & 0.1256 & $1.1256 \times 10^{-3}$ & 48.20 & $3 \times 10^{-3}$ & \\
\hline
\end{tabular}

The experimental data, which are shown in fig. 1 and in table 1, coincide with the calculations using Eq. (6) to within the accuracy of the measurements [4] - \pm 0.02 at. \%.

So it is possible to calculate the main composition of II-VI crystals and its variation along the crystal length. At constant temperature the quasistationary concentration is determined by ratio of the evaporation fluxes (flows), which depend only weakly on the applied pressure. The estimated composition of the crystals corresponds to the quasistationary one. In real growth conditions $(v \leq 4 \mu \mathrm{m} / \mathrm{s})$, the crystal composition is close to quasistationary value throughout most (up to $70 \%$ ) of the crystal length.

We see that in the general case of II-VI compounds and HPVB growth conditions:

(1) The composition of the melt (and crystal) shifts to the excess of the component with lower diffusion coefficient of vapors in the inert gas;

(2) The growth of crystals with stoichiometric composition throughout the entire length of the ingot or along most part of it is impossible.

\subsection{The HPVZM growth (in an inert gas atmosphere) of binary II-VI compound crystal in a graphite crucible}

In the case of HPVZM the composition changes due to:

1. Diffusion of the component vapours through crucible walls into the inert gas;

2. Crystallization of the melt;

3. Feeding of the zone by melting of an initial load.

Let's assume that:

- the width of the melted zone, its volume and the surface of evaporation are constants;

- diffusion in the solid phase and evaporation from its surface are negligible;

- the mixing in the melt is complete;

- the equilibrium of the gas phase with the melt is achieved instantly;

- segregation coefficient of the excessive component is constant and does not depend on concentration;

- the equilibrium at the boundary between the gas and liquid phases is achieved instantly;

- the $\mathrm{B}_{n} \leftrightarrow n \mathrm{~B}$ equilibrium in the gas phase is achieved instantly;

- external pressure of the inert gas is much more than the II-VI vapour pressure above the melt;

- the metal and chalcogen vapor pressure on the external surface of the crucible is equal to zero due to effective removing by convective flow of the inert gas with subsequent deposition on the cooled parts of the growth device.

Considering the aforementioned assumptions, the changing of the excessive component concentration in the melt is given by 


$$
\frac{d C_{i}}{d x}=-\left(\frac{\Pi_{i} S_{l} V_{m}}{V_{l} v_{p}}\right)-\left(\frac{k_{i}}{l}\right) \times C_{i}+\frac{C_{0, i}}{l}
$$

where $S_{l}$ is the area of the evaporation surface, $V_{l}$ is the melted zone volume; $x$ is the the distance covered by zone moving; $v_{p}$ is the rate of the zone moving; $\Pi_{i}$ is the difference between evaporation fluxes of metal and chalcogen; $l$ is the zone length.

In the right part of Eq. (7) the first member describes the concentration change due to evaporation from the melt surface, the second one is responsible for crystallization of the melt, and the last one is for the zone feeding.

The difference between evaporation fluxes of metal and chalcogen is given by:

$$
\Pi_{i}=\delta\left(p N_{B_{2}^{V I}}+q N_{A^{I I}}\right)
$$

where $p=2, q=-1$ in case of $\mathrm{B}^{\mathrm{VI}}$ excess in the melt and $p=-2, q=1$ in case of $\mathrm{A}^{\mathrm{II}}$ excess. Here, $N_{i}$ is the diffusion flux of the ith component.

Considering (7) it is possible to determine $\Pi_{i}$ as

$$
\Pi_{i}=\delta D_{i}\left(a P_{i}-b K_{0}^{b} D P_{i}^{m}\right) R T \Delta
$$

where $P_{i}$ is the vapor pressure of the $i$ th component at the temperature T; $K_{0}=P_{A^{I I}} \sqrt{P B_{2}^{V I}}$ is the constant of equilibrium of vapors with condensed phase at $T ; \mathrm{a}=2, \mathrm{~b}=1, \mathrm{~m}=-1 / 2, D=D_{A^{I I}} / D_{B_{2}^{V I}}$ in the case of $\mathrm{B}^{\mathrm{VI}}$ excess in the melt and $\mathrm{a}=1, \mathrm{~b}=2, \mathrm{~m}=-2$, and $D=D_{B_{2}^{V I}} / D_{A^{I I}}$ in the case of $\mathrm{A}^{\mathrm{II}}$ excess.

As we consider the solution of the $i$ th component in the melt to be perfect, the following expression allows and estimation of the $P_{i}$ value:

$$
P_{i}=\left(1-C_{i}\right) P_{0, i}+C_{i} P_{0, i}^{0} \approx P_{0, i}+C_{i} P_{0, i}^{0}
$$

where $C_{i}$ is the concentration in atomic fractions.

The HPVZM process consists of following three stages:

I. The holding of the first zone.

For this case the zone stays without moving during the time $\tau$. Changes in the excessive component concentration appear only due to diffusion through the crucible walls. The concentration changes from its initial value in the material $C_{0, i}$ to some intermediate one $C_{y, i}$, which will in turn be the starting concentration for the next HPVZM stage:

$$
\frac{d C_{i}}{d \tau}=-\frac{\prod_{i} S_{l} V_{m}}{V_{l}}
$$

The changing of the excessive component concentration can be determined through the changing of the component partial pressure above the melt. From Eq. (11) and taking into account Eqs. (8) and (9), we have the following expression:

$$
\frac{d P_{i}}{d \tau}=-\frac{\delta D_{i} S_{l} V_{m} P_{0, i}^{0}\left(a P_{i}-b K_{0}^{b} D P_{i}^{m}\right)}{R T V_{l} \Delta}
$$

The solution of (12) is:

$$
P_{i\left(C_{y, i}\right)}=\left[P_{i\left(C_{0, i}\right)}^{n} \times \exp \left(-\frac{3 P_{0, i}^{0} S_{l} V_{m} D_{i} \delta \tau}{V_{l} R T \Delta}\right)+c K_{0}^{b} D\left(1-\exp \left(-\frac{3 P_{0, i}^{0} S_{l} V_{m} D_{i} \delta \tau}{V_{l} R T \Delta}\right)\right)\right]^{h}
$$

where $n=3 / 2, c=1 / 2$, and $h=2 / 3$ in case of the chalcogen excess, and $n=3, c=2$, and $h=1 / 3$ in case of the metal excess. Here, $P_{i\left(C_{0, i}\right)}$ is the partial pressure of the $i t h$ component vapor at its start concentration, and $P_{i\left(C_{y, i}\right)}$ is the partial pressure of the $i$ th component vapor at its intermediate concentration.

\section{Zone moving.}


From the moment of the beginning of the zone moving, the changing in the excessive component concentration is due to evaporation, crystallization of the melt and zone feeding. The necessary equation can be obtained from Eqs. (7), (8) and (9):

$$
\frac{d P_{i}}{d x}=-\left(D_{i} \delta S_{l} V_{m} P_{0, i}^{0} / R T V_{l} v_{p} \Delta\right)\left(a P_{i}-b K_{0}^{b} D P_{i}^{m}\right)-\frac{k_{i} P_{i}}{l}+\frac{k_{i} P_{0, i}}{l}+\frac{P_{i\left(C_{0, i}\right)}-P_{0, i}}{l}
$$

On this stage the concentration changes from $C_{y, i}$ to the stationary one $C_{s t, i}$. After achieving of the stationary concentration $d P_{i} / d x=0$, and equation (14) has the following form:

$$
-\left(a A_{i}+k_{i} / l\right) P_{i\left(C_{s t, i}\right)}+A_{i} b D K_{0}^{b} P_{i\left(C_{s t, i}\right)}^{m}+\left\lfloor\left(k_{i}-1\right) P_{0, i}+P_{i\left(C_{0, i}\right)}\right\rfloor / l=0
$$

where $A_{i}=\left(D_{i} \delta S_{l} V_{m} P_{0, i}^{0} / R T V_{l} v_{p} \Delta\right)$. Here, $P_{i\left(C_{s t, i}\right)}$ is the partial pressure of the $i t h$ component vapor at its stationary concentration.

\section{Direct crystallization of the last zone.}

On this stage the conditions of crystallization is similar to HPVB. The concentration of the excessive component asymptotically decreases from the stationary value $C_{s t}$ to the quasi-stationary value, which is determined from the ratio of component evaporation fluxes by the $P_{A^{I I}} / P_{B_{2}^{V I}}=2 D_{B_{2}^{V I}} / D_{A^{I I}}$ condition.

The calculations of the stationary concentration of the excessive component show that it is determined mainly by two independent parameters - start concentration of the excessive component in the initial load and joint technological parameter (JTP) $l S_{l} / V_{l} v_{p}$. Fig. 2 shows the calculated dependences of the Zn stationary concentration in $\mathrm{ZnSe}$ as a function of the JTP value for different starting concentrations of zinc (HPVZM under 2.0 MPa pressure of Ar).

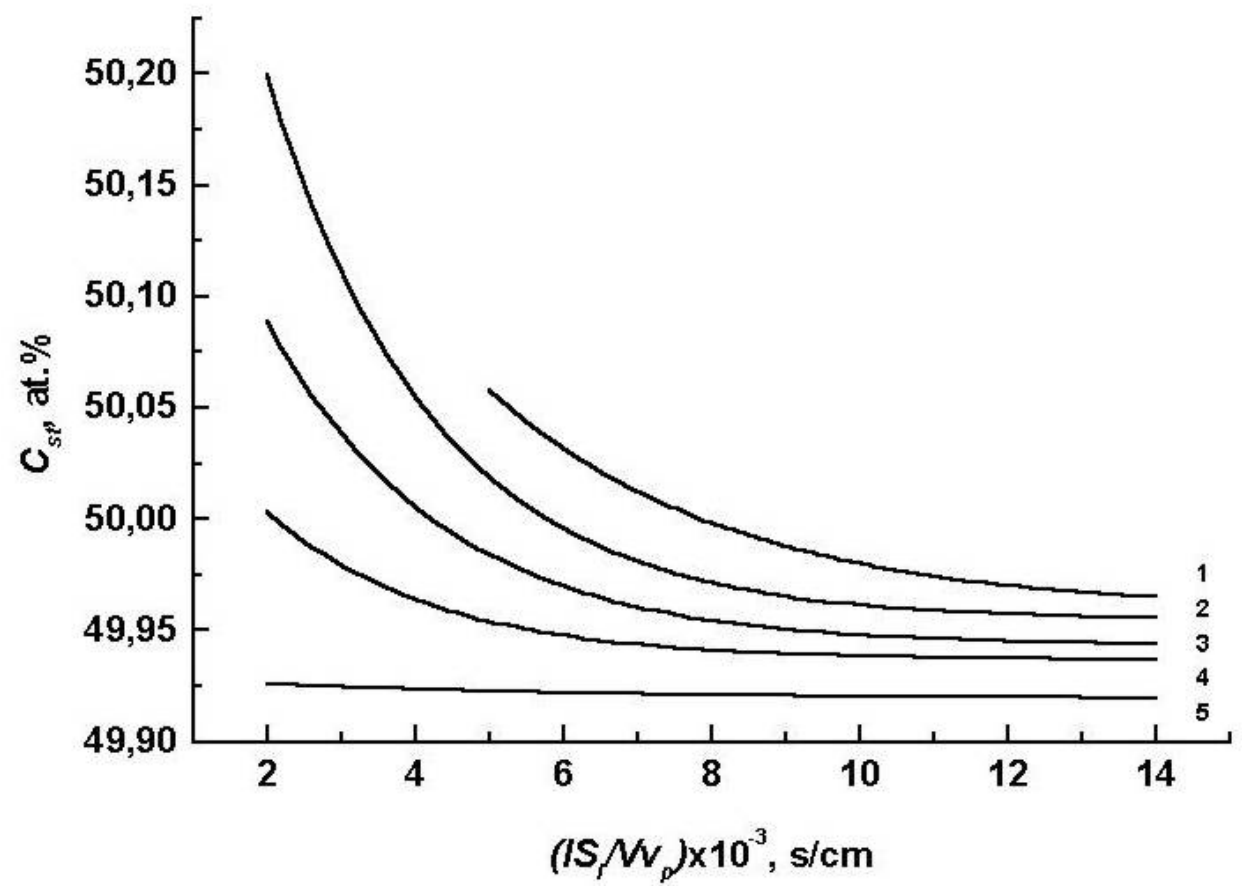

Fig. 2. Calculated dependences of the stationary Zn concentration in ZnSe on JTP as a function of starting Zn concentrations at 52.0,51.5, 51.0, 50.5 and 50.0 at. \% for curves 1, 2, 3, 4 and 5, correspondingly.

Fig. 3 shows the dependence of Zn stationary concentration in ZnSe on the starting Zn concentration at a fixed JTP value (HPVZM under 2.0 MPa pressure of Ar). The calculation coincides closely with the experimental data to within the accuracy of the composition analysis made by the method, as described in Ref. [4]. The data, shown on fig. 3 , 
represent the situation of HPVZM without holding of the first zone $(\tau=0)$. The segregation coefficient was assumed to be equal to 1 in the calculation.

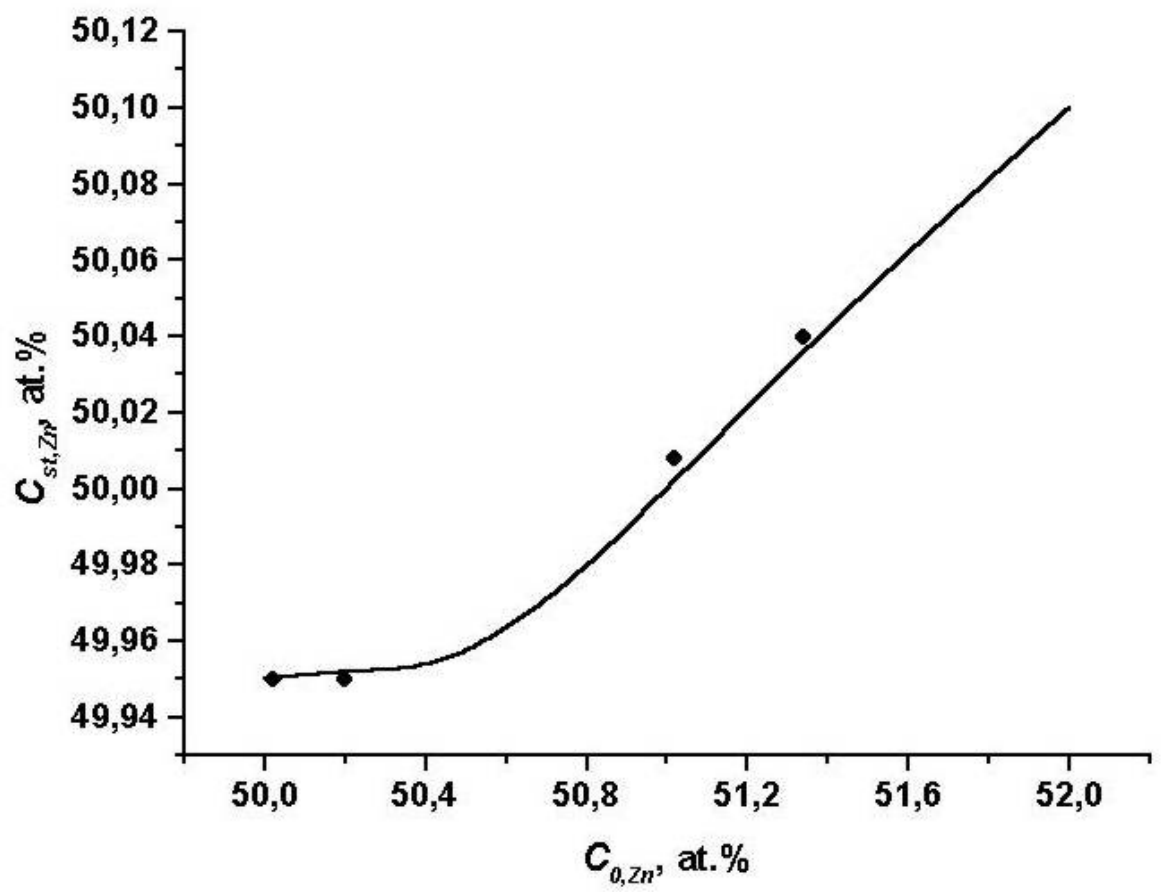

Fig. 3. Dependence of the stationary Zn concentration in ZnSe on its starting concentration at a fixed JTP value of $3.6 \times 10^{3}$ $\mathrm{s} / \mathrm{cm}$. The curve represents the calculated results, and the points are experimental data.

The calculations show that achieving stoichiometric stationary composition in II-VI crystals is possible only in the case when the initial load has an excess of the component with bigger diffusion coefficient of vapors in the inert gas.

The equations that describe the changing of the main composition during HPVZM of II-VI compound allow one to calculate the main composition shift from stoichiometry or to choose the technological parameters for growing crystals with preset main composition, including the stoichiometric one.

We were able to check the presented models for $\mathrm{ZnSe}$ and $\mathrm{ZnS}$ crystal growth and found that the approach is correct in all cases. It appeared that for $\mathrm{ZnS}$ the calculated quasistationary composition is equal to the stoichiometric one, so HPVZM has no serious advantages in case of the ZnS crystal growth. We did not apply the approach to CdS, $\mathrm{CdSe}, \mathrm{ZnTe}$ and CdTe due to the lack of the required data on their material properties. It is possible that the models are not exactly suitable for $\mathrm{CdTe}$, because they are based on the assumption that $\mathrm{B}^{\mathrm{VI}}$ component always evaporates in form of biatomic molecules. CdTe has the lowest melting point among binary zinc and cadmium chalcogenides $\left(1092{ }^{\circ} \mathrm{C}\right)$, and it is supposed that at this temperature in the vapor phase there may exist a perceptible amount of tellurium molecules with more than 2 atoms.

We consider that the same approach will be valid for ternary II-VI compounds too, but it is necessary to point out that the presented models describe the main composition of binary compounds only and thus are not directly applicable for ternary II-VI mixtures.

\section{SHAPED CRYSTAL GROWTH OF ZnSe, CdSe AND CdZnTe}

For the crystal growth of the ZnSe, CdSe and CdZnTe plates (tapes), we applied the device described in [5] with modifications according to [6-8]. ZnSe (fig. 4) and CdZnTe (fig. 5) tapes were grown without seed; CdSe plates (fig. 6) were grown on the oriented seed, and the applied orientations are shown in fig. 7. 


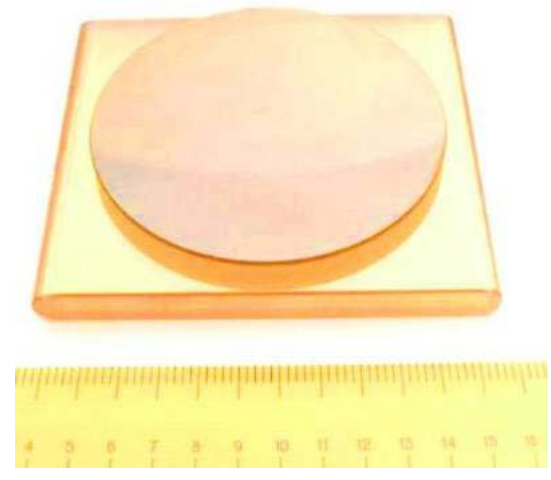

Fig. 4. ZnSe crystal tape (polished) and the lens manufactured from such tape (placed on top).

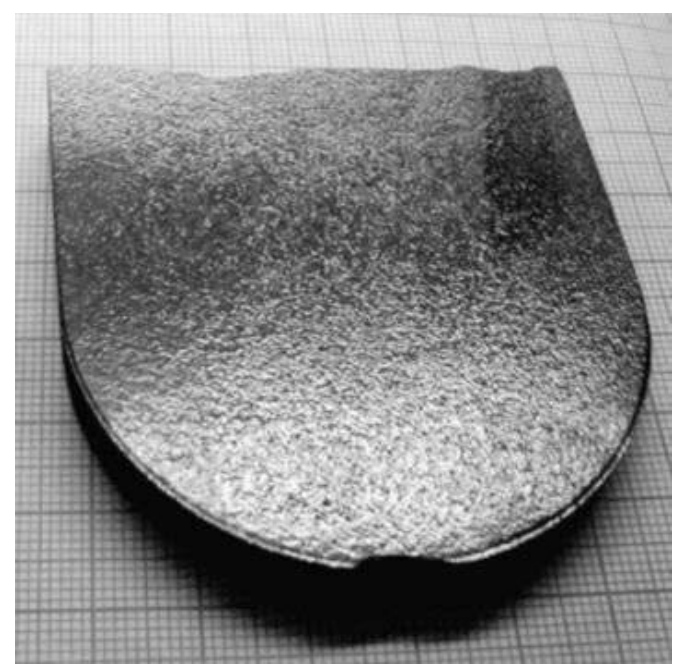

Fig. 6. CdSe single crystal plate ("as grown"). The scale is in $\mathrm{cm}$.

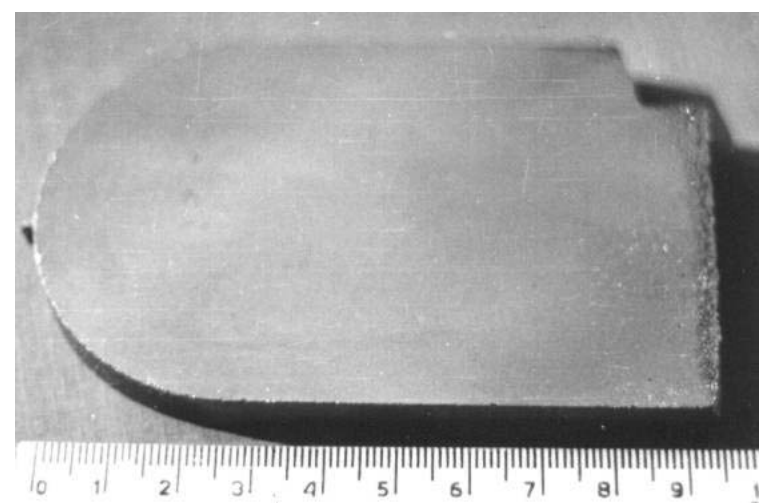

Fig. 5. $\mathrm{Cd}_{0.8} \mathrm{Zn}_{0.2}$ Te tape (lapped). The scale is in $\mathrm{cm}$.

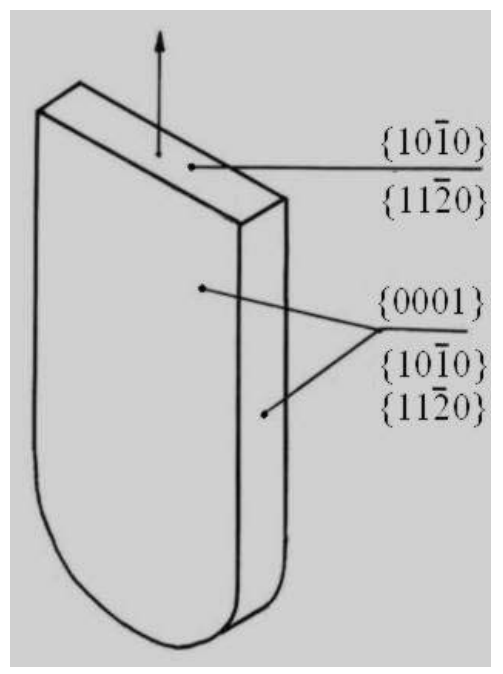

Fig. 7. Possible orientations of the CdSe single crystal tapes.

The growth device allows to carry out HPVB or HPVZM processes depending on the heater type.

The experimental results obtained in the HPVB and HPVZM growth run series are:

- Main composition of the crystal tapes corresponds to the models described in section 2 (the analysis was carried out only for $\mathrm{ZnSe}$ );

- The HPVZM allows to increase the tape volume approximately twice in comparison with the HPVB in the same device, i. e., without increasing of the internal sizes of the apparatus;

- The yield of the tapes, produced by HPVB, is approximately two times lower $(\approx 35 \%$ instead of $\approx 75 \%)$ in comparison with one for the HPVB grown cylindrical crystals. This is because of the heat field asymmetry in the rectangular heating zone;

- The yield of the HPVZM grown tapes is even lower than that of the HPVB ones $(\approx 25 \%)$, probably due to higher residual thermal stresses; 
- $\quad$ The tapes, grown without seed, are always polycrystals. I. e., growth of $\mathrm{ZnSe}$ (and $\mathrm{ZnS}$ ) single crystal tapes by HPVZM is impossible. ZnSe and $\mathrm{ZnS}$ undergo the wurtzite - sphalerite structural transition below the melting point $\left(\mathrm{ZnSe}\right.$ at $1420{ }^{\circ} \mathrm{C}, \mathrm{ZnS}$ at $\left.1020^{\circ} \mathrm{C}\right)$, so the growth on the oriented seed is impractical;

- All attempts to provide a convex crystallization front in the tape growth, both by HPVB and HPVZM, were unsuccessful. The front always had the concave form in both vertical cross-sections of the tape. As a result, the bubbles, formed in the melt, were not driven off by the front to the melt edge as in the case of the cylindrical II-VI compound crystal growth. Instead, the bubbles were driven to the center of the melt column and captured by the growing tape as shown on the photos fig. $8(\mathrm{a}, \mathrm{b})$ and $9(\mathrm{a}, \mathrm{b})$. (The problem of the bubbles will be discussed below in the section 4).
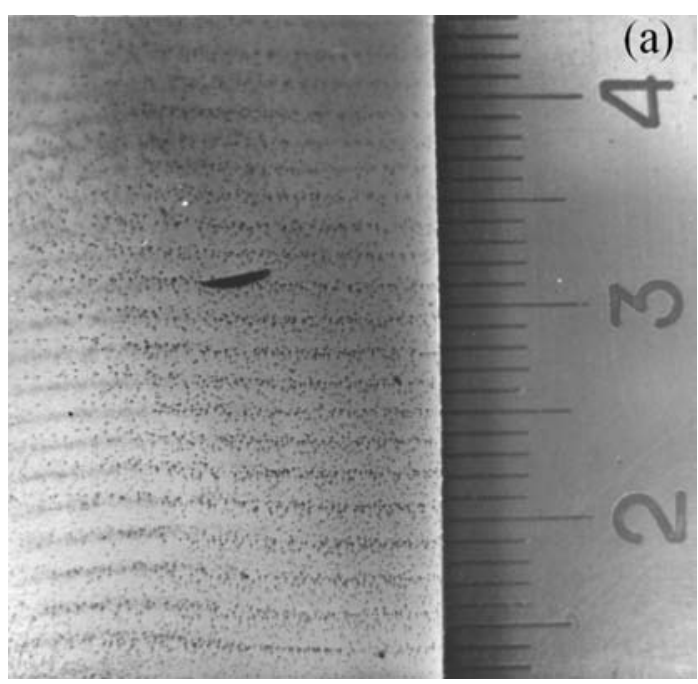

Fig. 8. Bubbles in the part of wide cross-section of the $\mathrm{ZnSe}$ tape (a) and in the narrow cross-section (b). The length scale is in $\mathrm{cm}$.

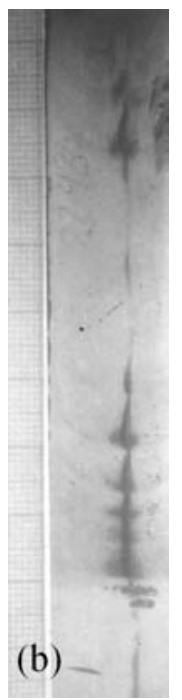

Fig. 9. Magnified "channel-like" (a) and "negative crystal" (b) bubbles in the ZnSe tape.

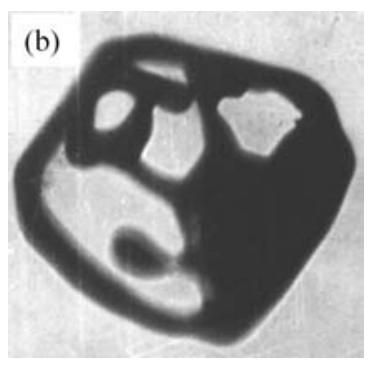

Table 2 represents some properties of the $\mathrm{ZnSe}$, CdSe and CdZnTe crystal tapes.

Table 2. Properties of the crystal tapes.

\begin{tabular}{|c|c|c|c|c|c|}
\hline $\begin{array}{c}\text { II-VI } \\
\text { compound }\end{array}$ & $\begin{array}{c}\text { Grown under } \\
\text { Ar pressure, } \\
\text { MPa }\end{array}$ & $\begin{array}{c}\text { Specific } \\
\text { resistivity, } \\
\text { Ohm } \times \mathbf{c m}\end{array}$ & $\begin{array}{c}\text { Conductivity } \\
\text { type }\end{array}$ & $\begin{array}{c}\text { IR transmittance } \\
\text { at 10.6 } \mu \mathbf{m}, \\
\mathbf{\%} / \mathbf{c m}\end{array}$ & $\begin{array}{c}\text { Absorption } \\
\text { coefficient at } \\
\mathbf{1 0 . 6} \boldsymbol{\mu m} \mathbf{c m}^{-\mathbf{1}}\end{array}$ \\
\hline $\begin{array}{c}\mathrm{ZnSe} \\
\text { "as grown" }\end{array}$ & 2 & $\geq 10^{11}$ & $n$ & 70.5 & $(1 \div 3) \times 10^{-3}$ \\
\hline $\begin{array}{c}\text { CdSe } \\
\text { as grown" }\end{array}$ & 2 & $(1 \div 3) \times 10^{2}$ & $n$ & - & - \\
\hline $\begin{array}{c}\text { CdSe } \\
\text { annealed in Se } \\
\text { vapours as in [9] }\end{array}$ & 2 & $\geq 10^{11}$ & $n$ & 71.0 & $1 \times 10^{-3}$ \\
\hline $\begin{array}{c}\mathrm{Cd}{ }_{0.8} \mathrm{Zn}_{0.2} \mathrm{Te} \\
\text { "as grown" }\end{array}$ & 2 & $\leq 10^{3}$ & $n$ & - & - \\
\hline $\mathrm{Cd}_{0.9} \mathrm{Zn}_{0.1} \mathrm{Te}$ & 10 & $1 \times 10^{8} \div 5 \times 10^{10}$ & $n^{*}$ & $58.0-62.0$ & - \\
\hline
\end{tabular}




\begin{tabular}{|c|c|c|c|c|c|}
\hline "as grown" & & & & & \\
\hline $\begin{array}{c}\mathrm{Cd}_{0.96} \mathrm{Zn}_{0.04} \mathrm{Te} \\
\text { "as grown" }\end{array}$ & 10 & $(6 \div 9) \times 10^{9}$ & $n^{*}$ & 64.0 & - \\
\hline
\end{tabular}

* - CdZnTe tapes were grown with intentional Zn doping to ensure obtaining $n$-type crystals.

\section{GROWTH OF BUBBLE-FREE TAPES}

We consider that the solubility of the inert gases in II-VI melts is very low. E. g., solubility of Ar in the ZnSe melt at $1530{ }^{\circ} \mathrm{C}$ under 2.0 MPa pressure, calculated according to [10] with the data on the melt properties from [3], is only $1.7 \times 10^{-6} \mathrm{~mol} / \mathrm{cm}^{3}$. Hence it is safe to assume that the bubbles in II-VI melts are filled with $\mathrm{A}^{\mathrm{II}}$ or $\mathrm{B}^{\mathrm{VI}}$ vapors due to the permanent dissociation of the melt. To corroborate this assumption the following set of experiments was carried out. Thirteen ZnSe crystal plates were grown with different stationary compositions, namely, with stoichiometric and with preset deviations of $\mathrm{Zn}$ and Se excess. Then the volume content of bubbles (Q) was measured for each plate by the difference of the X-ray determined density and the density, calculated from the results of hydrostatic weighting. The contribution of the vacancies and other defects to the density difference was considered negligible in comparison with that due to bubbles. The results of this experimental set are shown in fig. 10. It is easy to see that the bubble content linearly depends on the excess of both of the components. In crystals with selenium excess the Q value increases more rapidly than in the plates with zinc excess. This is because at the ZnSe melting point the pressure of Se vapors (26 MPa) exceeds the $\mathrm{Zn}$ vapor pressure $(6.5 \mathrm{MPa})$. The experimental results also show that growing stoichiometric plates do not result in obtaining bubble-free material - there still are some bubbles $(\mathrm{Q} \approx 0.2$ vol. \% for $\mathrm{ZnSe}$ in given conditions $)$ due to the permanent dissociation of the melt.

As it was mentioned in the previous section, it proved impossible to form a convex crystallization front for the plates. We found another solution for the growth of bubble-free plates. Application of the asymmetrical heater allowed formation of the "oblique-concave" crystallization front, as is schematically shown in fig. 11(a). Fig. 11(b) shows the vertical narrow cross-section of the ZnSe plate grown with such a front. It is visible that the bubbles are gathered along one side of the crystal plate, driven here by the melt front. The bubble-rich zone is only a few millimeters wide and could be easily removed by grinding. The rest of the crystal volume is practically bubble-free.

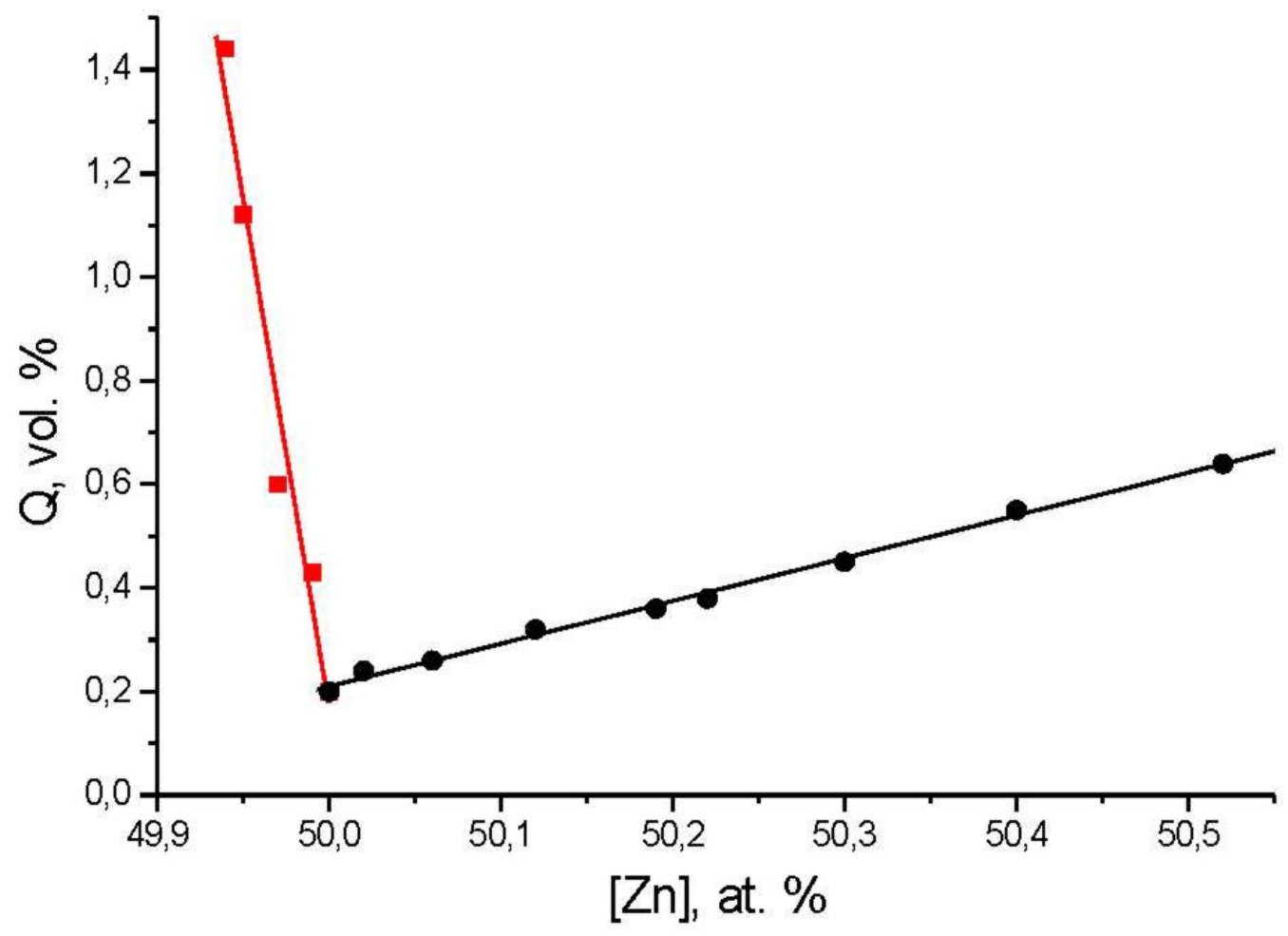


Fig. 10. Dependence of the volume content of the bubbles Q for HPVZM-grown ZnSe plates as a function their main composition (denoted in at. \% of zinc). The points represent experimental data, and the lines are the linear fit.

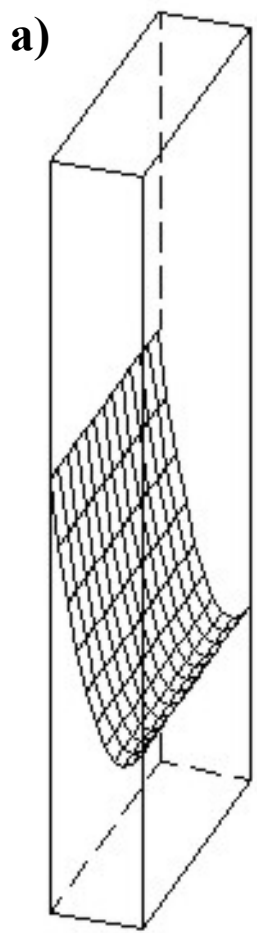

Fig. 11.

(a) - a schematic view of the "obliqueconcave" front;

(b) - part of the narrow cross-section of the ZnSe plate, grown with such front. The bubbles are gathered along the left side of the crystal plate.

The fine scale is in $\mathrm{mm}$.

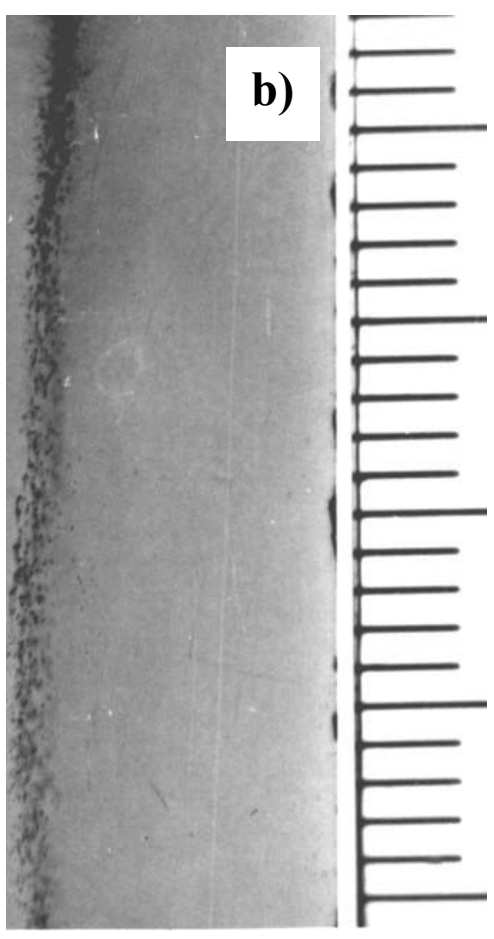

\section{CONCLUSIONS}

In general, HPVZM is preferable for crystal growth of II-VI compounds, because it allows control of the main composition and, hence, manufacturing of the crystals with stoichiometric composition or preset deviation from stoichiometry. At the same time the process presents some difficulties, because for successful HPVZM it is necessary to know a scope of properties, both of the II-VI compounds and their components over a wide range of temperatures and pressures. Currently, all the data necessary for HPVZM technological computations are available for only ZnSe. Nevertheless, the advantages of HPVZM for more stoichiometric growth are obvious and should lead to further investigation of II-VI compounds, which will gradually allow the extension of the method to other zinc and cadmium

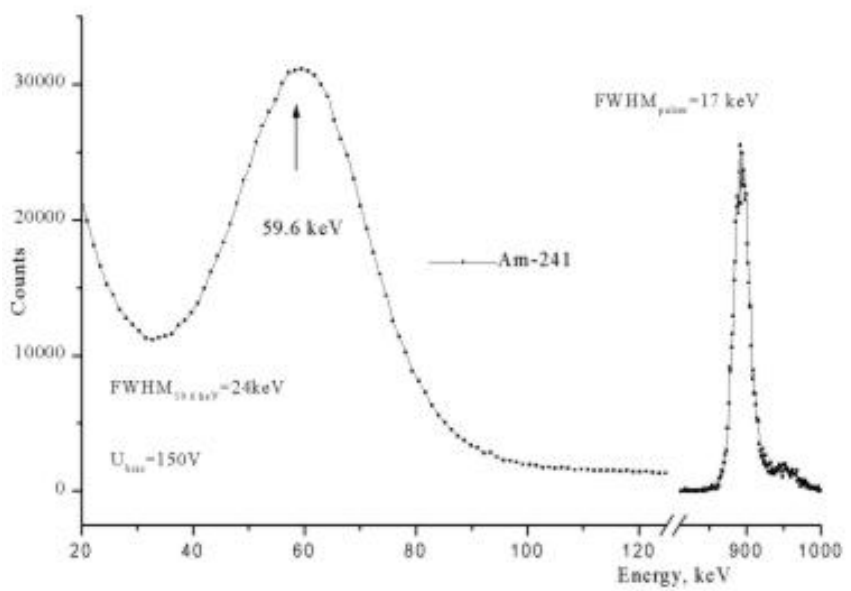


chalcogenides crystal preparation.

Fig. 12. Gamma (Am-241) energy spectrum of $10 \times 10 \times 2 \mathrm{~mm} \mathrm{Cd}_{0.9} \mathrm{Zn}_{0.1} \mathrm{Te}$ sample, cut from $60 \times 60 \times 6 \mathrm{~mm}$ tape.

Shaped growth of II-VI compound crystal plates (tapes) is useful in some cases, when the advantages outweigh the technical difficulties and the yields are acceptable. Such cases possibly are: CZT growth for large-volume ionizing radiation detectors, CdSe manufacturing for large diameter polarizers, and the preparation of big optical elements from ZnSe in situations where CVD-grade material is not suitable.

We successfully applied $\mathrm{ZnSe}$ tapes for manufacturing transfer, outlet and focusing optics for $\mathrm{CO}_{2}$ lasers. $\mathrm{ZnSe}$ windows, made from stoichiometric plates, worked over $1000 \mathrm{~h}$ for a $5 \mathrm{~kW}$ laser operating at a power density of 100 $\mathrm{kW} / \mathrm{cm}^{2}$. The elements from stoichiometric ZnSe were also used as multispectral optics (in visible - middle IR region).

Oriented CdSe tapes were applied for preparation of IR polarizers with diameters up to $60 \mathrm{~mm}$ and for manufacturing Wollaston prisms for the 10.6- $\mu \mathrm{m}$ wavelength application.

$10 \times 10 \times 2 \mathrm{~mm}$ samples from high-resistivity CZT tapes demonstrated suitable detector characteristics (see fig. 12), but we did not achieve such properties for the whole plates. All grown CZT tapes had inhomogeneous electrical properties. The reasons are not presently clear for the time being and further investigation is needed.

\section{ACKNOWLEDGEMENTS}

This work was partially supported by CRDF and the U.S. Department of Energy Initiatives for Proliferation Prevention Program. The authors are grateful to S. P. Vesnovskii and his co-workers from VNIIEF, Russia for measuring gamma-ray spectra for our CZT samples.

\section{REFERENCES}

1. N. N. Kolesnikov, M. P. Kulakov and A. V. Fadeev. "Changing of the ZnSe composition during zone melting," Izvestiya Akademii Nauk SSSR-Neorgan. mater., 22, N 3, p. 395, 1986.

2. M. P. Kulakov, A. V. Fadeev and N. N. Kolesnikov. "Determination of some properties of ZnSe melt and calculation of it composition,” Izvestiya Akademii Nauk SSSR-Neorgan. mater., 22, N 3, p. 399, 1986.

3. N. N. Kolesnikov, M. P. Kulakov and Yu. N. Ivanov. "Some properties of melts of $\mathrm{A}^{2} \mathrm{~B}^{6}$ compounds," J. Cryst. Growth, 125, p. 576, 1992.

4. A. V. Fadeev, M. P. Kulakov and N. N. Kolesnikov. "The method for analysis of Zn in ZnSe," Pat. USSR No $1171696,1983$.

5. M. P. Kulakov and A. V. Fadeev. "Device for crystal growth,” Pat. USSR No 813981, 1981.

6. N. N. Kolesnikov. "Crucible for II-VI compound crystal growth,” Pat. USSR No 1331132, 1985.

7. N. N. Kolesnikov and A. S. Kojevnikov. "Heater for crystal tape growth from the melt," Pat. USSR No 1140093, 1986.

8. N. N. Kolesnikov, M. P. Kulakov and V. I. Perminov. “Graphite heater,” Pat. USSR No 1656911, 1991.

9. N. N. Kolesnikov, M. P. Kulakov and V. K. Gartman. "Method of CdSe crystal processing," Pat. USSR No 1478690, 1987.

10. A. J. Appleby. "Solubility of non-interacting gases in molten salts," J. de Chimie-Physique et de Phys.-Chem. Biol., 74, p. 447, 1977. 